\title{
A Spatially Constrained Generative Model and an EM Algorithm for Image Segmentation
}

\author{
Aristeidis Diplaros, Associate Member, IEEE, Nikos Vlassis, and Theo Gevers, Member, IEEE
}

\begin{abstract}
In this paper, we present a novel spatially constrained generative model and an expectation-maximization (EM) algorithm for model-based image segmentation. The generative model assumes that the unobserved class labels of neighboring pixels in the image are generated by prior distributions with similar parameters, where similarity is defined by entropic quantities relating to the neighboring priors. In order to estimate model parameters from observations, we derive a spatially constrained EM algorithm that iteratively maximizes a lower bound on the data log-likelihood, where the penalty term is data-dependent. Our algorithm is very easy to implement and is similar to the standard EM algorithm for Gaussian mixtures with the main difference that the labels posteriors are "smoothed" over pixels between each Eand M-step by a standard image filter. Experiments on synthetic and real images show that our algorithm achieves competitive segmentation results compared to other Markov-based methods, and is in general faster.
\end{abstract}

Index Terms-Bound optimization, expectation-maximization (EM) algorithm, hidden Markov random fields (MRFs), image segmentation, spatial clustering.

\section{INTRODUCTION}

$\mathbf{I}$ $\mathrm{N}$ [1] and [2], Markov random field (MRF) models were introduced for image analysis. Subsequently, they have been used by many researchers for the solution of a number of important problems in image analysis such as image restoration, segmentation, edge-preserving filtering to name a few (see, e.g., [3]-[5] and references therein). MRF models provide a powerful and formal way to account for spatial dependencies between image pixels. A drawback of the aforementioned models is that it is typically very expensive to properly account for the pixels spatial dependencies during inference/learning. Various approximations have been introduced in order to make the problem tractable (e.g., multiresolution MRF [6]), but the high cost of MRF-based methods, as compared to other methods, still remains.

Manuscript received November 28, 2005; revised July 30, 2006; accepted September 28, 2006. This work is an Innovatiegerichte Onderzoeksprogramma (IOP) project supported by the Dutch Ministry of Economic Affairs under Project IBV99001.

A. Diplaros and T. Gevers are with the Informatics Institute and with the Intelligent Sensory Information Systems, Faculty of Science, University of Amsterdam, Amsterdam 1098SJ, The Netherlands (e-mail: diplaros@science.uva.nl; gevers@science.uva.nl).

$\mathrm{N}$. Vlassis was with the Informatics Institute and with the Intelligent Sensory Information Systems, Faculty of Science, University of Amsterdam, Amsterdam 1098SJ, The Netherlands. He is now with the Technical University of Crete, Hania, Crete 73100, Greece (e-mail: vlassis@science.uva.nl).

Color versions of one or more of the figures in this paper are available online at http://ieeexplore.ieee.org.

Digital Object Identifier 10.1109/TNN.2007.891190
In order to overcome this computational cost, several alternatives to MRF models have been proposed. These include modeling approaches that aim at directly defining hierarchical (Markovian) models on trees as in [7] and [8]. Also, Markov chains [9], [10] have been used, where the 2-D image is transformed into 1-D chain using some predefined sweep. These approaches, while being in general more computationally efficient compared to MRF, are less powerful in capturing spatial dependencies. In particular, as stated in [7], hierarchical models have a tendency to produce block-like artifacts in the final estimates. In [9], it is reported that Markov chains, while being more robust, tend to produce more irregular borders.

A particular problem that has been addressed by MRF models is image segmentation, the task of identifying homogeneous image regions or determining their boundaries. Formally, the task of image segmentation is to partition an image into a set of nonoverlapping regions $\left\{R_{1}, \ldots, R_{K}\right\}$, so that the variation of some property (such as intensity, color, texture, etc.) within each region $R_{k}$ is either constant or follows a simple model $\Phi_{k}$ (e.g., Gaussian) [11]. What makes this problem especially difficult is that the parameters for each model $\Phi_{k}$, as well as the corresponding regions $R_{k}$, have to be simultaneously estimated from the input image. To solve it, prior MRF models are commonly used in conjunction with iterative estimation procedures such as the expectation-maximization (EM) or other iterative algorithms [5].

In this paper, we introduce a novel generative model and an EM algorithm for Markov-based image segmentation. The proposed generative model assumes that the hidden class labels of the pixels are generated by prior distributions that share similar parameters for neighboring pixels. In order to define a notion of similarity between neighboring pixels priors, we introduce a pseudolikelihood quantity that couples neighboring priors by means of entropic quantities such as the KL divergence. To estimate the unknown parameters of the pixels prior distributions, as well as the parameters of the observation model, we derive an EM algorithm that iteratively maximizes an appropriately constructed lower bound on the data log-likelihood. The proposed algorithm is very similar to the standard EM algorithm for mixture models, with the main difference that the mixing weights (posterior distributions) of neighboring pixels are coupled in each EM iteration by an averaging operation. This results in a simple and efficient scheme for incorporating spatial constraints in an EM framework for image segmentation. Experimental results demonstrate the potential of the method on synthetic and real images.

The rest of this paper is organized as follows. In Section II, we briefly review the problem of image segmentation by discussing 
three classes of generative models that are commonly used in the literature. In Section III, we describe our proposed algorithm in detail and draw parallels with other existing approaches. In Section IV, we show experimental results and we conclude with a discussion in Section V.

\section{REVIEW OF MRF-BASED MODELS FOR IMAGE SEGMENTATION}

In this section, we discuss three commonly used probabilistic graphical models for image segmentation. The first one is a standard mixture model in which spatial dependencies between pixels are not explicitly incorporated into the generative model. The second one assumes that the hidden pixel labels form a Markov field. The third one, which is the one adopted in our method, assumes that the prior distributions that generate the pixel labels form a Markov field.

We first introduce the notation used throughout the paper. We are dealing with images consisting of $n$ pixels. For a pixel $i$, we denote by $y_{i}$ its observed value; for grayscale images, this is a scalar with values from 0 to 255 ; for color images this can be, e.g., a three-component vector with $R, G, B$ values. Moreover, we assume that each pixel $i$ belongs to a single class (image segment or region) which is indexed by the hidden random variable $x_{i}$. The latter takes values from a discrete set of labels $1, \ldots, K$. In all models we consider, we assume an observation model in the form $p\left(y_{i} \mid x_{i}\right)$ that describes the likelihood of observing $y_{i}$ given pixel label $x_{i}$. This model is a Gaussian ${ }^{1}$ density conditional on the class label $k$, i.e.,

$$
p\left(y_{i} \mid x_{i}=k, \theta\right)=\mathcal{N}\left(m_{k}, C_{k}\right)
$$

that is parameterized by its mean $m_{k}$ and (co)variance $C_{k}$, collectively denoted for all components by $\theta$. In all models we consider in this paper, the observation model is shared among all pixels, that is, the parameters $\theta=\left\{m_{k}, C_{k}\right\}_{k=1}^{K}$ are independent of the pixel index $i$.

\section{A. Standard Mixture Model}

This is the standard (Gaussian) mixture model [13] in which the spatial dependencies between pixels can be implicitly introduced by using the pixels coordinates as an extra feature [14]. This model is also employed in our previous work [15]. The corresponding generative model is shown in Fig. 1(a), where we show two neighboring pixels $i$ and $j$. The model assumes a common prior distribution $\pi$ that independently generates all pixel labels $x_{i}$. This prior $\pi$ is assumed to be a discrete distribution with $K$ states, whose parameters $\pi_{k}, k=1, \ldots, K$ are unknown, and it holds

$$
p\left(x_{i}=k \mid \pi\right)=\pi_{k}
$$

where we see that no spatial dependence between pixels is $a$ priori assumed (the prior $\pi_{k}$ has no dependence on pixel index $i$ ). Each pixel label $x_{i}$ generates a pixel observation $y_{i}$ from a shared Gaussian distribution $p\left(y_{i} \mid x_{i}, \theta\right)$ with parameters $\theta$ as

\footnotetext{
${ }^{1}$ This model cannot handle highly textured regions but there are alternatives (e.g., FRAME [12]) that can.
}

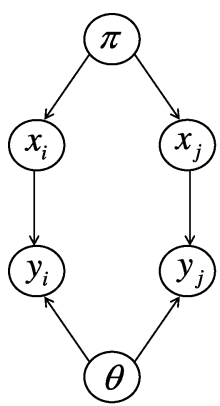

(a)

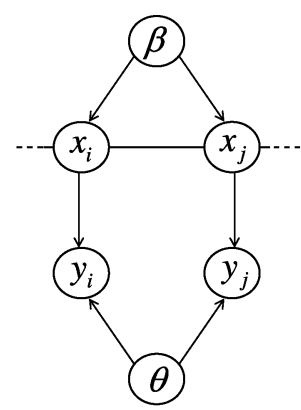

(b)

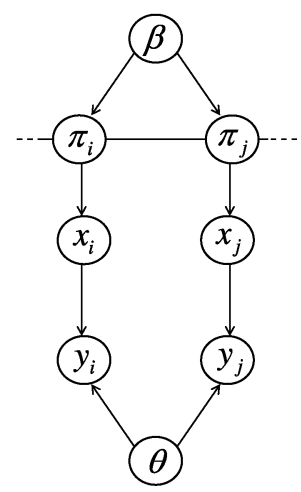

(c)
Fig. 1. Three commonly used probabilistic graphical models for image segmentation. Pixel $j$ is assumed to be in the neighborhood of pixel $i$. (a) Standard mixture model. (b) MRF on pixels labels. (c) MRF on pixel label priors.

described previously. The log-likelihood of all observations $y=$ $\left\{y_{1}, \ldots, y_{n}\right\}$ for the $n$ pixels is given by

$$
\begin{aligned}
\mathcal{L}_{1}(\theta, \pi) & =\sum_{i=1}^{n} \log \sum_{x_{i}=1}^{K} p\left(y_{i} \mid x_{i}, \theta\right) p\left(x_{i} \mid \pi\right) \\
& =\sum_{i=1}^{n} \log \sum_{k=1}^{K} p\left(y_{i} \mid x_{i}=k, \theta\right) \pi_{k} .
\end{aligned}
$$

The EM algorithm [16], [17] learns the parameters $\pi$ and $\theta$ by iteratively maximizing a lower bound of the log-likelihood $\mathcal{L}_{1}$. This bound is a function of the model parameters and a set of auxiliary distributions $q_{i}$

$$
\mathcal{F}_{1}\left(\theta, \pi,\left\{q_{i}\right\}\right)=\mathcal{L}_{1}(\theta, \pi)-\sum_{i} D\left(q_{i} \| p_{i}\right)
$$

where $D$ denotes the KL divergence between the two discrete distributions, which is defined as $D(A \| B)=$ $E_{A}[\log A-\log B]$, and which is always nonnegative and becomes zero when $A=B$. The distribution $p_{i} \equiv p\left(x_{i} \mid y_{i}\right)$ is the Bayes posterior of label $x_{i}$ given $y_{i}$ and parameters $\theta$ and $\pi$

$$
p\left(x_{i}=k \mid y_{i}\right)=\frac{p\left(y_{i} \mid x_{i}=k\right) \pi_{k}}{p\left(y_{i}\right)} .
$$

In the EM algorithm, we repeatedly maximize $\mathcal{F}_{1}$ over its parameters, in a coordinate ascent fashion. In the E-step, we fix $\theta$ and $\pi$ and optimize over $q_{i}$, and in the M-step, we fix $q_{i}$ and optimize over $\theta$ and $\pi$. This gives

$$
\begin{aligned}
\pi_{k} & =\frac{1}{n} \sum_{i=1}^{n} p\left(x_{i}=k \mid y_{i}\right) \\
m_{k} & =\frac{1}{n \pi_{k}} \sum_{i=1}^{n} p\left(x_{i}=k \mid y_{i}\right) y_{i} \\
C_{k} & =\frac{1}{n \pi_{k}} \sum_{i=1}^{n} p\left(x_{i}=k \mid y_{i}\right) y_{i} y_{i}^{\top}-m_{k} m_{k}^{\top} .
\end{aligned}
$$

We obtain similar equations in our algorithm which we will explain in detail in Section III. 


\section{B. MRF on Pixel Labels}

This model has been used, for instance, in [1] and [18]-[23], and is graphically shown in Fig. 1(b). Here, the vector of pixel labels $x=\left\{x_{1}, \ldots, x_{n}\right\}$ is assumed to be a (hidden) MRF with Gibbs joint probability distribution

$$
p(x \mid \beta)=\frac{1}{Z(\beta)} \exp (-H(x \mid \beta))
$$

where $H$ is an energy function

$$
H(x \mid \beta)=\sum_{C} V_{c}\left(x_{c} \mid \beta\right)
$$

parameterized by a set of clique potentials $V_{c}$ and some nonnegative scalar $\beta$. To deal with the inherent intractability of MRF [due to the normalizer in (10)], a standard approximation suggested by Besag [2], [24] and used, e.g., in [18] and [23] involves factorizing the joint distribution as

$$
p(x \mid \beta) \approx \prod_{i} p\left(x_{i} \mid x_{N_{i}}, \beta\right)
$$

where $N_{i}$ denotes the set of neighboring pixels of a pixel $i$. Using this approximation, the likelihood of the complete data (hidden pixel labels and pixel observations) reads

$$
p(y, x \mid \theta, \beta)=\prod_{i} p\left(y_{i} \mid x_{i}, \theta\right) p\left(x_{i} \mid x_{N_{i}}, \beta\right) .
$$

In particular, by clamping $x_{N_{i}}$ for each pixel $i$ to $\widetilde{x}_{N_{i}}$, the observed data log-likelihood becomes

$$
\mathcal{L}_{2}(\theta, \beta)=\sum_{i} \log \sum_{x_{i}=1}^{K} p\left(y_{i} \mid x_{i}, \theta\right) p\left(x_{i} \mid \widetilde{x}_{N_{i}}, \beta\right) .
$$

Note a similarity between $\mathcal{L}_{2}$ in (14) and $\mathcal{L}_{1}$ in (4); they are both mixture likelihoods with a parameter vector $\theta$ in the observation model that is shared by all pixels. Their difference is that the prior $\pi$ in $\mathcal{L}_{1}$ is shared by all pixels, whereas the prior $p\left(x_{i} \mid \widetilde{x}_{N_{i}}, \beta\right)$ in $\mathcal{L}_{2}$ is different for each pixel $i$ and depends on the neighbors $N_{i}$ of the pixel and the parameter $\beta$. We refer the reader to [18] for more details.

Maximizing $\mathcal{L}_{2}$ with respect to $\theta$ and $\beta$ can be carried out by the EM algorithm. In [23], for instance, each EM iteration involves a mean-field-like procedure in which the label $\widetilde{x}_{i}$ of a pixel $i$ is sequentially estimated from the values of its neighboring pixels $N_{i}$ as, e.g.,

$$
\widetilde{x}_{i}=\arg \max _{x_{i}} p\left(x_{i} \mid y_{i}, \widetilde{x}_{N_{i}}, \theta, \beta\right)
$$

where $p\left(x_{i} \mid y_{i}, \widetilde{x}_{N_{i}}, \theta, \beta\right)$ is the Bayes posterior given the parameters $\theta$ and $\beta$ of the previous iteration, and $\widetilde{x}_{N_{i}}$ includes a mix of previous and current estimated values (with respect to the current sweep over pixels). For each EM iteration, the previous procedure effectively requires computing a complete image restoration. We refer the reader to [23] for more details.

\section{MRF on Pixel Label Priors}

This is the model that we adopt in this paper, and which has also been used in [9], [11], [25], and [26]. It is graphically shown in Fig. 1(c). Here, the pixel label priors $\pi=\left\{\pi_{1}, \ldots, \pi_{n}\right\}$ are treated as random variables that form an MRF, whereas the pixel labels $x_{i}$ are assumed conditionally independent given the priors. In [25], the random field of the priors is defined as

$$
p(\pi \mid \beta)=\frac{1}{Z(\beta)} \exp (-U(\pi \mid \beta))
$$

where $U$ is an energy function in the form

$$
U(\pi \mid \beta)=\beta \sum_{i} \sum_{j \in N_{i}} \sum_{k=1}^{K}\left(\pi_{i k}-\pi_{j k}\right)^{2}
$$

parameterized by a scalar $\beta$. In the previous notation, $\pi_{i k}$ refers to the component $k$ of the prior distribution of pixel $i$. In this model, the priors $\left\{\pi_{i}\right\}$ are estimated together with $\theta$ by the EM algorithm. Translating the conditional independencies induced by the previous graphical model, the penalized log-likelihood of the observed data reads (ignoring constants)

$$
\begin{aligned}
\mathcal{L}_{3}(\theta, \pi)=\sum_{i} \log \sum_{k=1}^{K} p\left(y_{i} \mid x_{i}=k, \theta\right) \pi_{i k} & \\
& \quad-\beta \sum_{i} \sum_{j \in N_{i}} \sum_{k=1}^{K}\left(\pi_{i k}-\pi_{j k}\right)^{2} .
\end{aligned}
$$

We note the similarity of $\mathcal{L}_{3}$ with the $\mathcal{L}_{1}$ of the standard mixture model. Here, however, there are $n$ different $\pi_{i}$ distributions, one for each pixel $i$, and additionally there is a penalty term (the energy $U$ ) that penalizes neighboring pixels with different labels. Note that this model enforces spatial dependencies between pixels in a different way than the MRF model of Section II-B. Namely, here the assumption is that neighboring pixels have similar prior distributions that generate their pixel labels, whereas in the classical MRF model, we postulate an MRF directly on the pixel labels. An attractive property of this model, as we explain later, is that the E-step of the EM algorithm is easier to carry out since we do not need to estimate a restoration of the image. On the other hand, the M-step is more complicated as it also involves the penalty term $-U(\pi)$ of (16). Indeed, the computational effort in [11], [25], and [26] goes in the estimation of the $\left\{\pi_{i}\right\}$ priors in the M-step which requires solving a constrained optimization problem (since $\pi_{i}$ is a discrete distribution with $\sum_{k} \pi_{i k}=1$ for each $i$ ).

The main motivation for using this model as opposed to the traditional MRF model on pixel labels (of Section II-B) is its flexibility with respect to the initial conditions. This flexibility is manifested in the shape of the objective function. In the traditional MRF model of Section II-B, the penalized log-likelihood function will be sharper and will contain several local maxima, and hence, it will be more sensitive to the initial solution. In the current model, the field constraints are directly enforced over the parameters of the label priors, resulting in a smoother objective function. This can be intuitively seen by noting that distinct parameter values for some priors may induce exactly the 
same pixel labels, and therefore, searching in the space of priors (in the M-step of our algorithm as we show later) will be easier than searching in the space of labels. This search will be also facilitated by the fact that the space of prior parameters is continuous, as opposed to the discrete nature of the space of pixel labels. Interestingly, although the current model contains more parameters ( $K n$, a vector of $K$ parameters for each $\pi_{i}$, as opposed to $n$ of the traditional MRF model), the smoothness of the objective function, as argued previously, allows the result to be less dependent on the initialization. The previous arguments will be experimentally verified in Section IV.

\section{PROPOSED METHOD}

In our method, we also use the graphical model of Fig. 1(c), but we use a different modeling strategy for the spatial dependencies between the priors and a different algorithm for learning the unknown parameters. As in [23], we employ the Besag approximation for modeling the joint density over pixel priors

$$
p(\pi \mid \beta) \approx \prod_{i} p\left(\pi_{i} \mid \pi_{N_{i}}, \beta\right)
$$

where we define $\pi_{N_{i}}$ as a mixture distribution over the priors of neighboring pixels of pixel $i$, i.e.,

$$
\pi_{N_{i}}=\sum_{\substack{j \in N_{i} \\ j \neq i}} \lambda_{i j} \pi_{j}
$$

where $\lambda_{i j}$ are fixed positive weights and for each $i, \sum_{j} \lambda_{i j}=1$ holds. The mixing weight $\lambda_{i j}$ depends on the relative offset between the pixels $i$ and $j$, while the mixture does not include the prior of the same pixel $i$. Note that the evaluation of this mixture corresponds to a convolution operation $\pi_{. k} * \lambda$, for each component $k$, where $\lambda$ is a symmetric linear image filter with zero coefficient in its center and nonnegative coefficients elsewhere that sum to one. See Section IV-B for more details about filter related issues.

Further, for the conditional density $p\left(\pi_{i} \mid \pi_{N_{i}}, \beta\right)$, we assume an approximate log-model in the form (ignoring constants)

$$
\log p\left(\pi_{i} \mid \pi_{N_{i}}, \beta\right)=-\beta\left[D\left(\pi_{i}|| \pi_{N_{i}}\right)+H\left(\pi_{i}\right)\right]
$$

where $D\left(\pi_{i} \| \pi_{N_{i}}\right)=\sum_{k=1}^{K} \pi_{i k} \log \pi_{i k}-\sum_{k=1}^{K} \pi_{i k} \log \pi_{N_{i} k}$ is the KL divergence between $\pi_{i}$ and $\pi_{N_{i}}$, which is always nonnegative and becomes zero when $\pi_{i}=\pi_{N_{i}}$, and $H\left(\pi_{i}\right)=$ $-\sum_{k=1}^{K} \pi_{i k} \log \pi_{i k}$ is the entropy of the distribution $\pi_{i}$, which is always nonnegative and reaches its maximum when $\pi_{i}$ is uniform. The KL term $D\left(\pi_{i} \| \pi_{N_{i}}\right)$ intuitively expresses the degree of similarity between the prior of a pixel $i$ and the priors of its neighbors, and it provides a way of constraining neighboring pixels to have similar class labels. Similarly, the entropy term $H\left(\pi_{i}\right)$ constrains the label priors to be as informative as possible: in homogeneous regions it is reasonable to expect that neighboring pixels have similar priors and that these priors are far from uniform. It is important to emphasize that the entropy term $H\left(\pi_{i}\right)$ does not enforce the priors $\pi_{i}$ to be of a particular shape, but it merely constrains them to be as informative as possible.
Maximum a posteriori (MAP) estimation of the parameters $\theta$ and $\pi_{i}$ of our model involves maximizing the data log-likelihood [the first term of $\mathcal{L}_{3}$ in (18)] penalized by the approximate log-prior term (21). Direct optimization of this penalized log-likelihood is, however, difficult because of the coupling of neighboring pixels priors $\pi_{i}$ (which would require constraint optimization techniques as those in [26]). To facilitate optimization, we introduce an approximation that makes use of an auxiliary set of distributions $s_{i}$ as follows:

$$
\log p\left(\pi_{i} \mid \pi_{N_{i}}, \beta, s_{i}\right) \approx-\beta\left[D\left(s_{i} \| \pi_{i}\right)+D\left(s_{i} \| \pi_{N_{i}}\right)+H\left(s_{i}\right)\right]
$$

which decouples the pixels priors and allows for an efficient coordinate ascent EM-like optimization as we will show later. Note that in the previous approximation, when $s_{i}=\pi_{i}$, then (22) becomes identical to (21).

The previous approximate log-prior involves only entropic quantities and therefore is a nonnegative quantity that lower bounds the data log-likelihood. Note that this penalty term (Bayesian prior) does not depend on the observed data (image pixels). Recently, other approaches have appeared in the machine learning literature that incorporate constraints into a learning problem by lower bounding the data log-likelihood using data-dependent penalty terms [27], [28]. Typically, those bounds involve a KL distance between posterior distributions, thus explicitly incorporating the observed data into the bounding terms, and utilizing useful domain knowledge. In the same spirit, we introduce an additional penalty term involving posterior distributions in the form

$$
-\frac{1}{2}\left[D\left(q_{i} \| p_{i}\right)+D\left(q_{i} \| p_{N_{i}}\right)+H\left(q_{i}\right)\right]
$$

where $q_{i}$ is an arbitrary class distribution for a pixel $i$, and $p_{i}$ is the posterior class distribution of a pixel $i$ computed for model parameters $\theta$ and prior $\pi_{i}$ by the Bayes rule

$$
p_{i k} \equiv p\left(x_{i}=k \mid y_{i}, \theta, \pi_{i k}\right)=\frac{p\left(y_{i} \mid x_{i}=k, \theta\right) \pi_{i k}}{\sum_{l=1}^{K} p\left(y_{i} \mid x_{i}=l, \theta\right) \pi_{i l}} .
$$

The coefficient $1 / 2$ in the penalty term was chosen because it allows a tractable M-step. Putting all terms together, the penalized log-likelihood of the observed data as a function of the model parameters and the introduced auxiliary distributions $s=\left\{s_{i}\right\}$ and $q=\left\{q_{i}\right\}$ reads (ignoring constants)

$$
\begin{aligned}
\mathcal{F}(\theta, \pi, s, q) & \\
=\sum_{i}[ & \log \sum_{k} p\left(y_{i} \mid x_{i}=k, \theta\right) \pi_{i k} \\
& -\frac{1}{2}\left[D\left(q_{i} \| p_{i}\right)+D\left(q_{i} \| p_{N_{i}}\right)+H\left(q_{i}\right)\right] \\
& \left.-\beta\left[D\left(s_{i} \| \pi_{i}\right)+D\left(s_{i} \| \pi_{N_{i}}\right)+H\left(s_{i}\right)\right]\right] .
\end{aligned}
$$

Our EM algorithm maximizes the energy $\mathcal{F}$ in (25) by coordinate ascent. In the E-step, we fix $\theta$ and $\pi$ and maximize $\mathcal{F}$ over $s$ and $q$. In the M-step, we fix $s$ and $q$ and maximize $\mathcal{F}$ over $\theta$ and $\pi$. Next, we show how these two steps can be performed. 
E-Step: We optimize over $s_{i}$ for a pixel $i$, assuming $\theta$ and $\pi$ fixed. A similar derivation holds for $q_{i}$. The terms of $\mathcal{F}$ involving $s_{i}$ are

$$
\begin{aligned}
& -\sum_{k} s_{i k} \log s_{i k}+\sum_{k} s_{i k} \log \pi_{i k}-\sum_{k} s_{i k} \log s_{i k} \\
& +\sum_{k} s_{i k} \log \pi_{N_{i} k}+\sum_{k} s_{i k} \log s_{i k} \\
& \quad=-\sum_{k} s_{i k} \log s_{i k}+\sum_{k} s_{i k} \log \left(\pi_{i k} \pi_{N_{i} k}\right) .
\end{aligned}
$$

The latter is an (unnormalized) negative KL-divergence which becomes zero when

$$
s_{i} \propto \pi_{i} \pi_{N_{i}} .
$$

Similarly, we obtain

$$
q_{i} \propto p_{i} p_{N_{i}}
$$

M-Step: We fix $s$ and $q$ and maximize $\mathcal{F}$ over $\theta$ and $\pi$. The terms of $\mathcal{F}$ involving the priors $\pi_{i}$ and the posteriors $p_{i}$ (and, therefore, $\theta$ and $\pi_{i}$ ) are

$$
\begin{array}{r}
\log \sum_{k} p\left(y_{i} \mid x_{i}=k, \theta\right) \pi_{i k}-\frac{1}{2}\left[D\left(q_{i} \| p_{i}\right)+\sum_{\substack{j \in N_{i} \\
j \neq i}} D\left(q_{j} \| p_{N_{j}}\right)\right] \\
-\beta\left[D\left(s_{i} \mid \pi_{i}\right)+\sum_{\substack{j \in N_{i} \\
j \neq i}} D\left(s_{j} \| \pi_{N_{j}}\right)\right] . \quad \text { (29) }
\end{array}
$$

We show now the derivation involving the posteriors. The terms of (29) involving only $p_{i}$ are

$$
-\frac{1}{2}\left[D\left(q_{i} \| p_{i}\right)+\sum_{\substack{j \in N_{i} \\ j \neq i}} D\left(q_{j} \| p_{N_{j}}\right)\right]
$$

which, ignoring terms independent of $p_{i}$, reads

$$
-\frac{1}{2}\left[-\sum_{k} q_{i k} \log p_{i k}-\sum_{\substack{j \in N_{i} \\ j \neq i}} \sum_{k} q_{j k} \log p_{N_{j} k}\right]
$$

where

$$
p_{N_{j}}=\sum_{\substack{m \in N_{j} \\ m \neq j}} \lambda_{j m} p_{m}=\lambda_{j i} p_{i}+\sum_{\substack{m \in N_{j} \\ m \neq i, j}} \lambda_{j m} p_{m} .
$$

The mixture $p_{N_{j}}$ appears in the last term of (31) for all pixels $j$ that are neighbors of a pixel $i$. To make the M-step tractable, we bound these terms using Jensen's inequality

$$
\begin{aligned}
\log p_{N_{j} k} & =\log \sum_{\substack{m \in N_{j} \\
m \neq j}} \lambda_{j m} p_{m k} \\
& \geq \lambda_{j i} \log p_{i k}+\sum_{\substack{m \in N_{j} \\
m \neq i, j}} \lambda_{j m} \log p_{m k}
\end{aligned}
$$

Using (31) and (33) and noting that $\lambda_{j i}=\lambda_{i j}$, we finally get (ignoring again terms independent of $p_{i}$ )

$$
\begin{array}{r}
\frac{1}{2}\left[\sum_{k} q_{i k} \log p_{i k}+\sum_{k} \sum_{\substack{j \in N_{i} \\
j \neq i}} \lambda_{j i} q_{j k} \log p_{i k}\right] \\
\Rightarrow \frac{1}{2} \sum_{k}\left(q_{i k}+q_{N_{i} k}\right) \log p_{i k}
\end{array}
$$

where the distribution $q_{N_{i}}$ is

$$
q_{N_{i}}=\sum_{\substack{j \in N_{i} \\ j \neq i}} \lambda_{i j} q_{j}
$$

An identical derivation holds for the priors producing a term

$$
\beta \sum_{k}\left(s_{i k}+s_{N_{i} k}\right) \log \pi_{i k} .
$$

In total, the terms of $\mathcal{F}$ [actually a lower bound of it since we employed (33)] involving the prior $\pi_{i}$ and the posterior $p_{i}$ are

$$
\begin{aligned}
\log \sum_{k} p\left(y_{i} \mid x_{i}=k, \theta\right) \pi_{i k} & +\frac{1}{2} \sum_{k}\left(q_{i k}+q_{N_{i} k}\right) \log p_{i k} \\
& +\beta \sum_{k}\left(s_{i k}+s_{N_{i} k}\right) \log \pi_{i k} .
\end{aligned}
$$

Expanding the posterior $p_{i}$ in the previous terms and noting that $(1 / 2) \sum_{k}\left(q_{i k}+q_{N_{i} k}\right)=1$, we immediately see that the loglikelihood term cancels. Then, (37) reads

$$
\begin{aligned}
& \frac{1}{2} \sum_{k}\left(q_{i k}+q_{N_{i} k}\right) \log p\left(y_{i} \mid x_{i}=k, \theta\right) \pi_{i k} \\
& +\beta \sum_{k}\left(s_{i k}+s_{N_{i} k}\right) \log \pi_{i k} \\
& \Rightarrow \frac{1}{2} \sum_{k}\left(q_{i k}+q_{N_{i} k}\right) \log p\left(y_{i} \mid x_{i}=k, \theta\right) \\
& \quad+\sum_{k}\left[\frac{1}{2}\left(q_{i k}+q_{N_{i} k}\right)+\beta\left(s_{i k}+s_{N_{i} k}\right)\right] \log \pi_{i k} .
\end{aligned}
$$

Collecting all terms of (38) involving $\pi_{i}$ and differentiating with respect to $\pi_{i}$ (using a Lagrange multiplier to ensure $\sum_{k} \pi_{i k}=$ 1 ), we can easily show that we get

$$
\pi_{i}=\frac{1}{(1+2 \beta)}\left[\frac{1}{2}\left(q_{i}+q_{N_{i}}\right)+\beta\left(s_{i}+s_{N_{i}}\right)\right] .
$$

Similarly, differentiating (38) over $\theta$, we get the following update equations for the means and covariances of the $K$ Gaussian components:

$$
\begin{aligned}
m_{k} & =\frac{\sum_{i}\left(q_{i k}+q_{N_{i} k}\right) y_{i}}{\sum_{i}\left(q_{i k}+q_{N_{i} k}\right)} \\
C_{k} & =\frac{\sum_{i}\left(q_{i k}+q_{N_{i} k}\right) y_{i} y_{i}^{\top}}{\sum_{i}\left(q_{i k}+q_{N_{i} k}\right)}-m_{k} m_{k}^{\top} .
\end{aligned}
$$




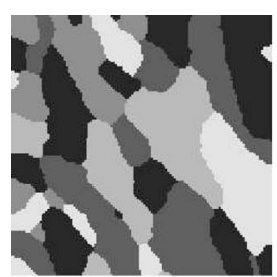

(a)

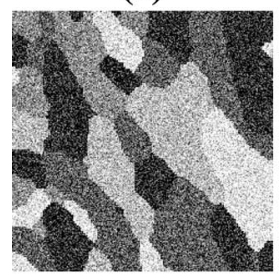

(c)

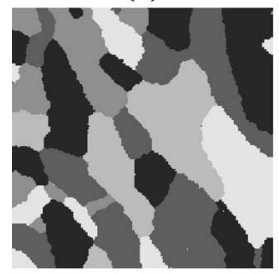

(f)

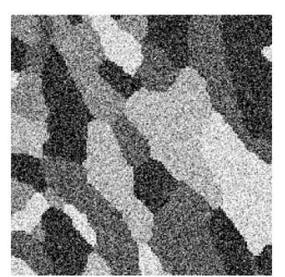

(d)

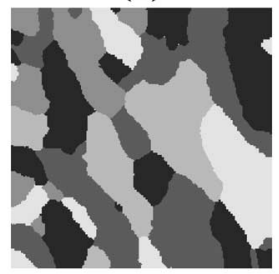

(g)

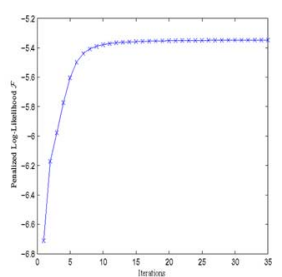

(b)

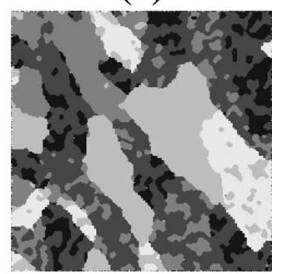

(e)

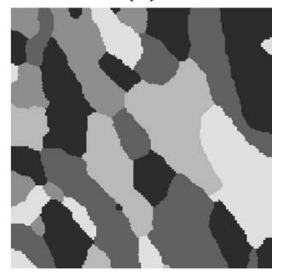

(h)
Fig. 2. Experiment with synthetic image and white Gaussian noise. (a) Fiveclass synthetic image. (b) Maximization progress of the penalized log-likelihood $\mathcal{F}$ of our algorithm for this experiment. (c) Noise corrupted five-class image with additive white Gaussian noise $(\sigma=52)$. (d) Segmentation result of the standard EM algorithm (MCR 53.7\%). (e) Segmentation result of ICM with running time of $39 \mathrm{~s}$ (MCR 31.7\%). (f) Segmentation result of SimF with running time of $90 \mathrm{~s}$ (MCR 2.88\%). (g) Segmentation result of MeanF with running time of $100 \mathrm{~s}$ (MCR 3.89\%). (h) Segmentation result of our approach with running time of $92 \mathrm{~s}$ (MCR 1.78\%).

Note that the update equations for $\theta$ are analogous to those in the standard mixture model, with the difference that here the weights correspond to "smoothed" pixel posteriors. The use of such spatially smoothed weights in the M-step of the EM algorithm is a key element in our approach that distinguishes it from other works. The complete algorithm is shown in Algorithm 1.

Concerning the initialization of the parameter vector $\theta$, we employ the $k$-means algorithm, but we note that other clustering algorithms [29] can be used also. The initialization of the priors $\left\{\pi_{i}\right\}$ in this work is uniform. Concerning time complexity, each EM step has cost that is linear in the number of pixels in the image and linear in the number of class labels, as we can directly see in the EM update equations, for instance, (27) and (40). Additionally, our update equations involve a convolution operation for computing the "smoothed" distributions $\pi_{N_{i}}, p_{N_{i}}, s_{N_{i}}$, and $q_{N_{i}}$, which, for each pixel $i$, has constant runtime complexity (since the size of the filter is fixed). Concerning the convergence rate of our algorithm, we have experimentally observed that our method can quickly reach a good solution indicated by high values of $\mathcal{F}$ [see Figs. 2(b) and 3(b)]. This is in accordance with similar findings for the batch EM algorithm in the literature [17], but for which theoretical evidence is, to our knowledge, still lacking.

Finally, in this paper, we set $\beta=0.5$. It is a matter of future work to investigate ways to incorporate $\beta$ in the optimization process (as in [22] and [23]).

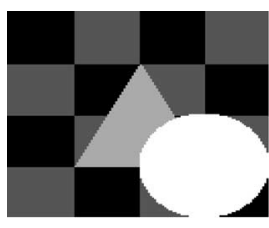

(a)

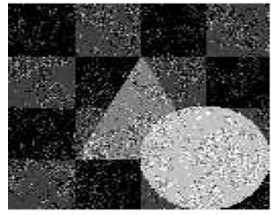

(c)

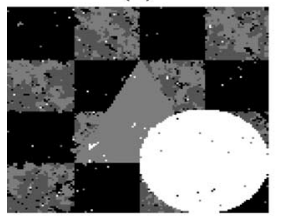

(f)

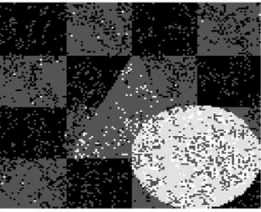

(d)

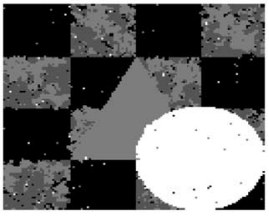

(g)

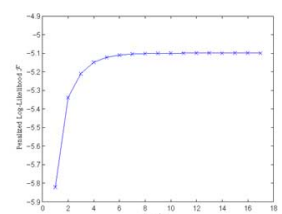

(b)

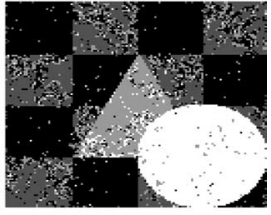

(e)

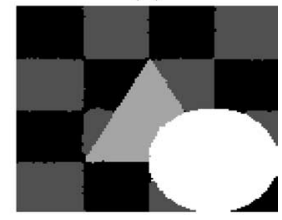

(h)
Fig. 3. Experiment with synthetic image and spatially correlated Gaussian noise. (a) Original four-class image. (b) Maximization progress of the penalized $\log$-likelihood $\mathcal{F}$ of our algorithm for this experiment. (c) Noise-corrupted image with spatially correlated Gaussian noise. (d) Segmentation result of the standard EM algorithm (MCR 28.72\%). (e) Segmentation result of ICM with running time of $5 \mathrm{~s}$ (MCR 16.8\%). (f) Segmentation result of SimF with running time of $22 \mathrm{~s}$ (MCR 17.6\%). (g) Segmentation result of MeanF with running time of $23 \mathrm{~s}$ (MCR 17.4\%). (h) Segmentation result of our approach with running time of $6 \mathrm{~s}$ (MCR $0.68 \%$ ).

Algorithm 1: The proposed EM algorithm for image segmentation

1) Initialize the parameter vector $\theta$, e.g., using $k$-means.

2) Initialize the priors $\left\{\pi_{i}\right\}$, e.g., uniform $\pi_{i k}=(1 / K)$, $\forall i, k$.

3) E-step: Compute posterior probabilities $p_{i}$ using (24) and the current estimates of $\theta$ and $\left\{\pi_{i}\right\}$.

4) Compute $\left\{s_{i}\right\}$ according to (27) and (20) and normalize each $s_{i}$ so that $\sum_{k} s_{i k}=1$.

5) Compute $\left\{q_{i}\right\}$ according to (28) and normalize each $q_{i}$ so that $\sum_{k} q_{i k}=1$.

6) M-step: Update the parameter vector $\theta$ using (40) and (41).

7) Update $\left\{\pi_{i}\right\}$ according to (39).

8) Evaluate $\mathcal{F}$ from (25).

9) If convergence of $\mathcal{F}$, e.g., $\left|\mathcal{F}_{t+1} / \mathcal{F}_{t}-1\right|<\epsilon$

10) then stop.

11) else go to step 3 ).

12) end if

\section{EXPERIMENTS}

In this section, we demonstrate the performance of our algorithm on synthetic and real images. Specifically, in Section IV-A, we evaluate the performance of our segmentation algorithm in the presence of noise. Section IV-B includes additional experiments to test the algorithm's behavior with respect to the choice of parameters and initialization. Section IV-C 


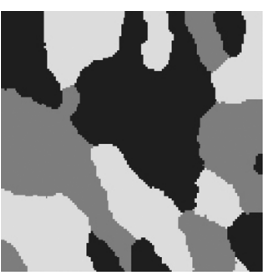

(a)

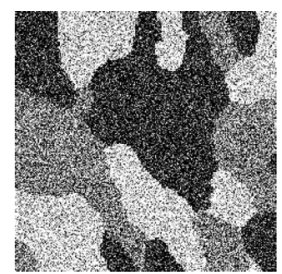

(b)

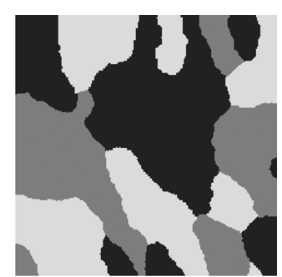

(c)
Fig. 4. Effects of using different filters on the segmentation results. (a) Threeclass synthetic image. (b) Noise-corrupted image with additive white Gaussian noise of $\sigma=95$. (c) Segmentation result using Gaussian kernel with $\sigma=1$ (MCR $1.08 \%$ ).

presents segmentation results on graylevel images. Finally, in Section IV-D, we show segmentation results on color images.

\section{A. Noise-Corrupted Synthetic Images}

We first illustrate our algorithm on synthetic images and consider its robustness against noise. We use the same synthetic images as in [20] and [26]. These are simulated three- and five-class images [see Figs. 4(a) and 2(a)] sampled from an MRF model using the Gibbs sampler [1]. In Fig. 2(c), we show the five-class image after adding white Gaussian noise with $\sigma=52$. In Fig. 2(d), we show the segmentation results of the standard EM algorithm using the generative model discussed in Section II-A. It is clear from these examples that, in presence of noise, an algorithm that does not use spatial constraints cannot produce meaningful segmentation results. On the contrary, a method like ours that does take into account the spatial relation of pixels can successfully segment these noisy images, as we demonstrate in Fig. 2(h). The total running time of our method (from initialization till convergence) was $15 \mathrm{~s}$ for the three-class image and $92 \mathrm{~s}$ for the five-class one [as shown in Fig. 2(h)]. All times (for our method) refer to a Matlab implementation running on a 3.0-GHz PC-based workstation. In these synthetic images, the ground truth is known (the true assignment of pixels to the $K$ classes), which allows us to evaluate the performance of the various methods in terms of the misclassification ratio (MCR). This is simply the number of misclassified pixels divided by the total number of pixels [20].

We compare our method with related methods based on the hidden MRFs: the ICM algorithm [2], the spatially variant finite mixture model (SVFMM) method [25], the extension to SVFMM (SVFMM-QD)[26], the hidden MRF model based on EM framework proposed in [20] (HMRF-EM), and the mean field (MeanF) and the simulated field (SimF) methods of [21] and [23]. Finally, we have also included the comparison with the standard EM algorithm.

Tables I and II contain the MCR results of the previously mentioned methods for the same synthetic images and for various amounts of noise. For the methods SVFMM, SVFMM-QD, and HMRF-EM, we replicate the MCR results reported in the corresponding papers. For SimF and MeanF methods, we used a software implementation developed in [21] and [23] (in C programming language) and is publicly available at http://mistis.inrialpes.fr/software/SEMMS.html. This software also includes an implementation of the ICM algorithm. We initialize ICM, SimF, and MeanF methods in the same way that we initialize our algorithm. As in [21] and [23], the number of iterations for SimF and MeanF was set to 100 and the ICM was run until the convergence.
TABLE I

MCRS FOR THE THREE-CLASS IMAGE

\begin{tabular}{|c|c|c|c|c|c|c|}
\hline & \multicolumn{6}{|c|}{ Noise } \\
\hline & 18 & 25 & 28 & 47 & 52 & 95 \\
\hline$\overline{\overline{E M}}$ & $0.61 \%$ & $3.64 \%$ & $5.82 \%$ & $20.5 \%$ & $23.9 \%$ & $42.3 \%$ \\
\hline HMRF-EM & - & - & $0.12 \%$ & $1.04 \%$ & - & $8.73 \%$ \\
\hline ICM & $0.03 \%$ & $0.15 \%$ & $0.21 \%$ & $0.81 \%$ & $0.96 \%$ & $4.01 \%$ \\
\hline SimF & $0.02 \%$ & $0.08 \%$ & $0.13 \%$ & $0.43 \%$ & $0.62 \%$ & $1.52 \%$ \\
\hline MeanF & $0.02 \%$ & $0.08 \%$ & $0.12 \%$ & $0.39 \%$ & $0.54 \%$ & $1.32 \%$ \\
\hline SVFMM & $0.2 \%$ & $1.5 \%$ & - & - & $21 \%$ & - \\
\hline SVFMM-QD & $0.1 \%$ & $1 \%$ & - & - & $13 \%$ & - \\
\hline Our Approach & $0.03 \%$ & $0.1 \%$ & $0.1 \%$ & $0.46 \%$ & $0.50 \%$ & $1.18 \%$ \\
\hline $\begin{array}{l}\text { run time of } \\
\text { our approach }\end{array}$ & $5.8 \mathrm{sec}$ & $7.7 \mathrm{sec}$ & $8.8 \mathrm{sec}$ & $14 \mathrm{sec}$ & $15 \mathrm{sec}$ & $29 \mathrm{sec}$ \\
\hline
\end{tabular}

TABLE II

MCRS FOR THE FIVE-CLASS IMAGE

\begin{tabular}{l||c|c|c|c|c|c}
\multicolumn{1}{l||}{} & \multicolumn{6}{c}{ Noise } \\
& 18 & 23 & 25 & 33 & 47 & 52 \\
\hline \hline EM & $15.1 \%$ & $27.1 \%$ & $28.2 \%$ & $43.1 \%$ & $53.8 \%$ & $53.7 \%$ \\
HMRF-EM & - & $0.2 \%$ & - & $1.36 \%$ & $7.68 \%$ & - \\
ICM & $0.47 \%$ & $0.94 \%$ & $1.24 \%$ & $4.44 \%$ & $24.6 \%$ & $31.7 \%$ \\
SimF & $0.26 \%$ & $0.46 \%$ & $0.47 \%$ & $0.92 \%$ & $2.19 \%$ & $2.88 \%$ \\
MeanF & $0.23 \%$ & $0.38 \%$ & $0.49 \%$ & $0.73 \%$ & $2.41 \%$ & $3.89 \%$ \\
SVFMM & $6 \%$ & - & $30 \%$ & - & - & $42 \%$ \\
SVFMM-QD & $4 \%$ & - & $10 \%$ & - & - & $28 \%$ \\
Our Approach & $0.24 \%$ & $0.39 \%$ & $0.49 \%$ & $0.73 \%$ & $1.49 \%$ & $1.78 \%$ \\
\hline run time of & & & & & & \\
our approach & $19 \mathrm{sec}$ & $23 \mathrm{sec}$ & $27 \mathrm{sec}$ & $40 \mathrm{sec}$ & $76 \mathrm{sec}$ & $92 \mathrm{sec}$ \\
\hline
\end{tabular}

For our method, the restorations shown result from the maximization of the estimated prior distribution $\pi_{i}$ (which the algorithm learns). The SVFMM, SVFMM-QD, and HMRF-EM methods use a first-order neighborhood system, while, ICM, SimF, and MeanF methods use a second-order neighborhood system.

In Fig. 3, we present the segmentation results for a synthetic four-class image in which we have added spatially correlated noise. To generate this noise, we sampled a configuration of binary indicators from a standard Potts-model MRF [1] using Gibbs sampling. If the drawn indicator of a pixel was one, then we added Gaussian noise with standard deviation $\sigma=52$ to the corresponding pixel, otherwise, we did not add noise. Clearly, this kind of noise invalidates the Gaussian observation model assumption used by all methods discussed in this paper (since the true observation model is now a Gaussian mixture), and renders the observations spatially correlated. Thus, it presents a case where the true generative model of the image is different than the postulated one, which makes it an interesting experiment for real-world data. In Fig. 3(c), we show the noise corrupted image. In Fig. 3(d)-(g), we present the segmentation results of the EM, ICM, SimF, and MeanF. Fig. 3(h) shows the segmentation obtained by our method. Clearly, we see an advantage of our method over the other methods on this problem. We note that we initialized all algorithms in the same way.

In Figs. 2(b) and 3(b), we present the maximization progress of the penalized $\log$-likelihood $\mathcal{F}$ for the simulated five-class image of Fig. 2 and the simulated five-class image of Fig. 3, respectively.

The experiments point out that in all methods the MCR increases as the amount of noise and the number of labels $K$ increase. Our method performs much better than all the other methods when moderate or high amount of noise is present, and it is competitive to other methods for low amounts of noise. 
TABLE III

COEFFICIENTS OF THE FILTER USED IN THE EXPERIMENTS

\begin{tabular}{|c|c|c|c|c|}
\hline 0 & 0.0185 & 0.0414 & 0.0185 & 0 \\
\hline 0.0185 & 0.0852 & 0.0865 & 0.0852 & 0.0185 \\
\hline 0.0414 & 0.0865 & 0 & 0.0865 & 0.0414 \\
\hline 0.0185 & 0.0852 & 0.0865 & 0.0852 & 0.0185 \\
\hline 0 & 0.0185 & 0.0414 & 0.0185 & 0 \\
\hline
\end{tabular}

TABLE IV

MCRS FOR Fig. 4(b) WITH DIFFERENT FILTERS

\begin{tabular}{|c|c|c|c|}
\hline Gaussian $(\sigma=0.5)$ & Gaussian $(\sigma=1)$ & mean $3 \times 3$ & mean $5 \times 5$ \\
\hline $1.19 \%$ & $1.08 \%$ & $1.23 \%$ & $1.16 \%$ \\
\hline
\end{tabular}

Our method, implemented in Matlab, was faster than SimF and MeanF methods, which were implemented in $\mathrm{C}$ and optimized. In Fig. 2, we present the running time and the segmentation results of ICM, SimF, and MeanF methods for the five-class image.

\section{B. Choice of Parameters}

In total, our algorithm has three parameters, the number of components $K$, the priors parameter $\beta$, and the image filter used in (20). In this paper, we assume that the number of components $K$ as well as the observation model family are given to us for a particular image. Also, in experiments not shown here, we determined that 0.5 is a good choice for the parameter $\beta$. As aforementioned, it is a matter of future work to investigate ways to incorporate this parameter in the optimization process. In this section, we will consider the only other free parameter in our algorithm which is the filter used for evaluating the mixtures $\pi_{N_{i}}, p_{N_{i}}, q_{N_{i}}$, and $s_{N_{i}}$ [see (20) and (35)].

Specifically, as mentioned in Section III, the evaluation of such mixtures can be achieved by convolving with an image filter. In order for these mixtures to be valid distributions (without the need of extra normalization), the applied filter must have coefficients that sum to one. Our algorithm also requires that the center coefficient of the filter be zero. Also, we choose the filter to be symmetric. In the case where domain knowledge would imply some (simple) spatial relation between pixels with the same class label, this knowledge could be easily incorporated into our framework by employing a nonsymmetric filter that encourages these spatial relations.

Clearly, the choice of the filter can affect the performance of the algorithm and the quality of the segmentation. In addition, the mixture calculation can be performed by a variety of images filters. In Section IV-A, all reported results of our algorithm were obtained using a "modified" (i.e., the center coefficient set to zero) pillbox filter with diameter $\delta$ equal to 5 . We show the coefficients of this filter in Table III.

In Table IV, we report the MCR results using different filters for the three-class image [see Fig. 4(a)], after adding white Gaussian noise with $\sigma=95$ [see Fig. 4(b)]. We used "modified" versions (i.e., the center coefficient is set to zero) of a low-pass Gaussian filter and a mean filter. In Fig. 4(c), we show the corresponding segmentation results for Gaussian filters with $\sigma=1$. The results illustrate that a larger filter size can compensate for the presence of high noise levels in the image. A closer look at Fig. 4(a) and (c) reveals that large size filters, while providing increased robustness to noise, tend to oversmooth the edges. When the noise levels in the image are low there is no benefit from

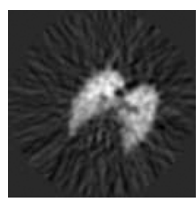

(a)

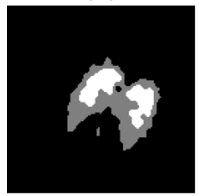

(c)

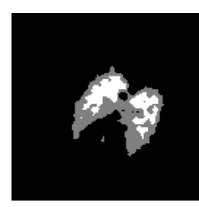

(b)

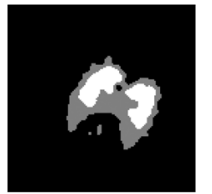

(d)

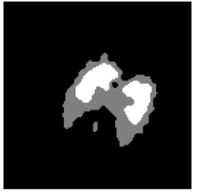

(e)

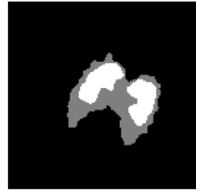

(f)
Fig. 5. Segmentation example of the PET image of a dog lung with $K=3$. (a) Original image. (b) Simple thresholding-based initialization. (c) Segmentation result of ICM. (d) Segmentation result of SimF. (e) Segmentation result of MeanF. (f) Segmentation result of our approach.

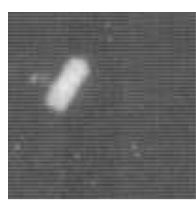

(a)

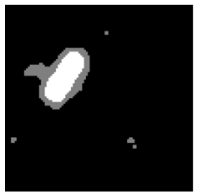

(d)

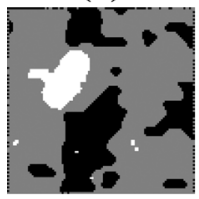

(h)

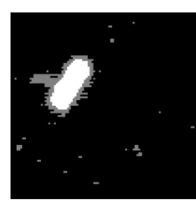

(b)

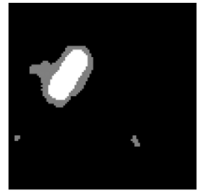

(e)

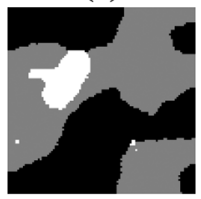

(i)

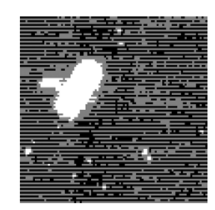

(c)

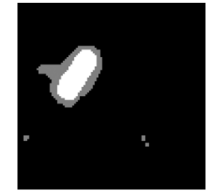

(f)

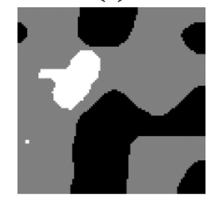

(j)

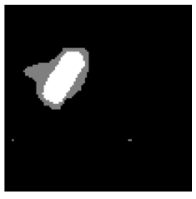

(g)

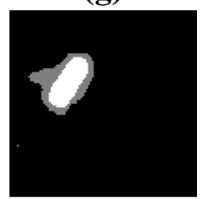

(k)
Fig. 6. Segmentation example of the buoy image with $K=3$. (a) Original image. (b) $k$-means initialization. (c) Initialization based on EM for independent mixtures. (d) Segmentation result of ICM with $k$-means initialization. (e) Segmentation result of SimF with $k$-means initialization. (f) Segmentation result of MeanF with $k$-means initialization. (g) Segmentation result of our approach with $k$-means initialization. (h) Segmentation result of ICM with EM initialization. (i) Segmentation result of SimF with EM initialization. (j) Segmentation result of MeanF with EM initialization. (k) Segmentation result of our approach with EM initialization.

using large size filters, and the resulting oversmoothing of the edges just decreases the segmentation accuracy. Clearly, if the noise level of images in a particular domain is known a priori, then the filter type and its parameters can be fine-tuned for optimal performance.

\section{Graylevel Images}

In natural images, a number of difficult aspects of image segmentation come together, such as noise and varying imaging conditions. Additionally, the true value of the image label $K$ is not known. In the following experiments, we use the same images as in [21], [30], and [31], where $K$ was estimated using (approximations of) the Bayesian information criterion (BIC). Fig. 5(a) shows a $128 \times 128$ positron emission tomography (PET) image of a dog lung and Fig. 6(a) shows an aerial 100 


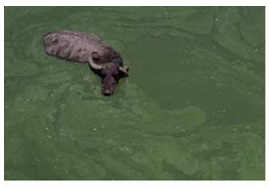

(a)

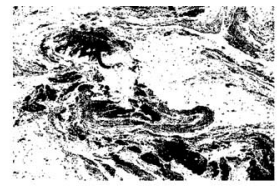

(b)

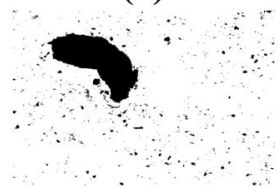

(d)

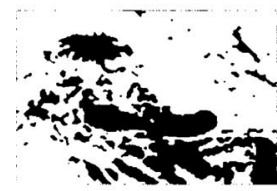

(f)

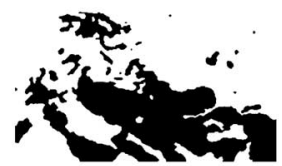

(g)

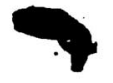

(j)

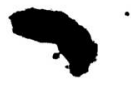

(c)

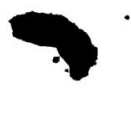

(e)

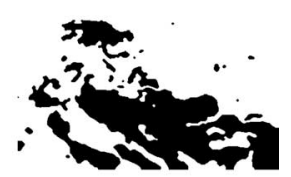

(h)

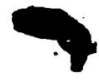

(k)

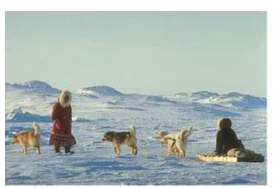

(a)

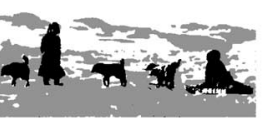

(f)

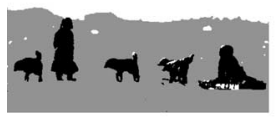

(i)

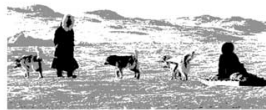

(b)

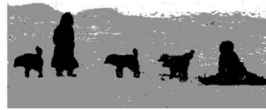

(d)

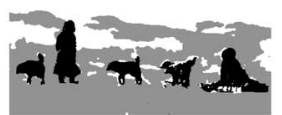

(g)

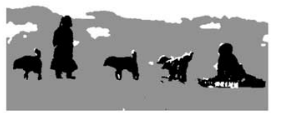

(j)

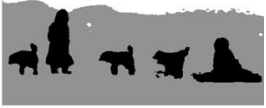

(c)

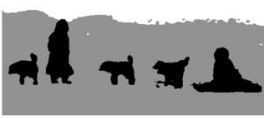

(e)

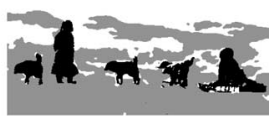

(h)

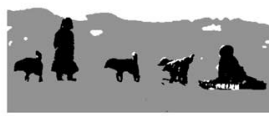

(k)
Fig. 7. Segmentation example of the water buffalo image with $K=2$. (a) Original image. (b) $k$-means initialization. (c) Segmentation result of our approach with $k$-means initialization. (d) Initialization based on EM for independent mixtures. (e) Segmentation result of our approach with EM initialization. (f) Segmentation result of ICM with $k$-means initialization. (g) Segmentation result of SimF with $k$-means initialization. (h) Segmentation result of MeanF with $k$-means initialization. (i) Segmentation result of ICM with EM initialization. (j) Segmentation result of SimF with EM initialization. (k) Segmentation result of MeanF with EM initialization.

$\times 100$ image of a buoy against a background of dark water (see [30] for more details on their nature and origin).

For the PET image of a dog lung, the image's labels $K$ was estimated to be 3. In Fig. 5(c)-(f), we demonstrate the segmentation results of ICM, MeanF, SimF, and our approach. For our method, we used a "modified" (i.e., the center coefficient set to zero) pillbox filter with diameter $\delta$ equal to 7 . All other settings for all methods were the same as in Section IV-A. All algorithms were initialized by a simple thresholding of the image, as shown in Fig. 5(b). The result shows that MeanF, SimF, and our approach perform approximately in the same way and are producing more homogeneous regions for the lungs than the ICM algorithm.

For the buoy image, the image's label $K$ was estimated to be 3. In Fig. 6(d)-(g), we demonstrate the segmentation results of ICM, MeanF, SimF, and our approach. We used the same settings for all methods as in Section IV-A. All algorithms were initialized using the $k$-means-based initialization which is shown in Fig. 6(b). It is clear that all algorithms given this particular initialization perform approximately the same and they are all capable of correctly assigning the pixels belonging to the buoy to one cluster. In Fig. 6(h)-(k), we show the segmentation results of the four methods using the same setting as before but employing an alternative initialization based on EM for independent mixtures. This initialization is shown in Fig. 6(c), where the horizontal scan lines from the imaging process of Fig. 6(a)
Fig. 8. Segmentation example of the dog sled image with $K=3$. (a) Original image. (b) $k$-means initialization. (c) Segmentation result of our approach with $k$-means initialization. (d) Initialization based on EM for independent mixtures. (e) Segmentation result of our approach with EM initialization. (f) Segmentation result of ICM with $k$-means initialization. (g) Segmentation result of SimF with $k$-means initialization. (h) Segmentation result of MeanF with $k$-means initialization. (i) Segmentation result of ICM with EM initialization. (j) Segmentation result of SimF with EM initialization. (k) Segmentation result of MeanF with EM initialization.

can be clearly observed. Only our approach was able to correctly assign the pixels belonging to the buoy to one cluster given this EM-based initialization.

The buoy experiment demonstrates a significant aspect of our approach, namely, that it is relatively insensitive to initialization compared to other methods. We feel that this is an important aspect, since in natural images, not only the true value of the image label $K$ is hard to estimate, but also an appropriate initialization cannot be known a priori.

\section{Color Images}

In Figs. 7 and 8, we show the segmentation results of two different color images from the Berkeley Segmentation Data Set [32]. In these figures, we show the original image, the initializations obtained by $k$-means and EM, the results of our method with these two initializations, and the corresponding results of ICM, SimF, and MeanF. In these examples, our algorithm is able to consistently segment the original image independently of the initialization, whereas all three MRF-based methods are rather sensitive to the initial conditions.

In Fig. 9, we show the segmentation results of an image in $r g b$ color space when the Gaussian observation model assumption is violated. Normalized $r g b$ color (chromaticity) has been widely used by many researchers in the field of image segmentation, e.g., [33] and [34], because of its important invariant properties. Specifically, it has been shown in [35] that, under the assumption of the dichromatic reflection model, normalized color is to 


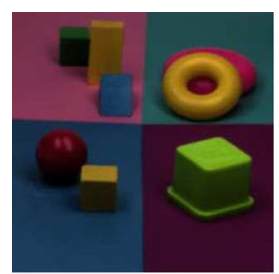

(a)

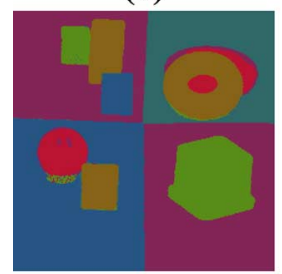

(d)

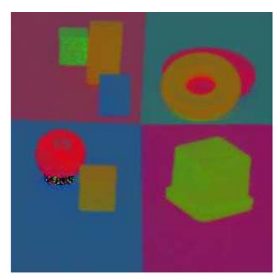

(b)

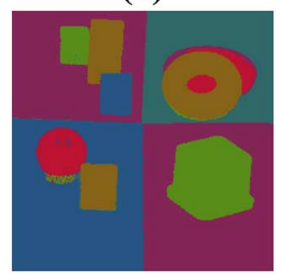

(e)

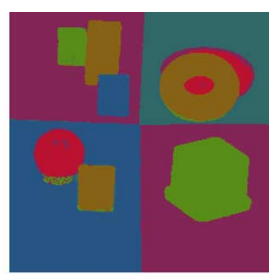

(c)

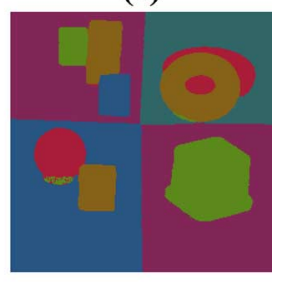

(f)
Fig. 9. Segmentation example of a color image with $K=6$. (a) Original image. (b) Normalized $r g b$ color image. (c) Segmentation result of ICM in $r g b$ color space. (d) Segmentation result of SimF in $r g b$ color space. (e) Segmentation result of MeanF in $r g b$ color space. (f) Segmentation result of our approach in $r g b$ color space.

a large extent invariant to a change in camera viewpoint, object pose, and the direction and intensity of the incident light. In addition, the color transformation from $R G B$ to normalized color $r g b$ is simple and easy to compute while does not necessitate extra color-reduction steps, as in [36]. Namely, based on the measured $R G B$ values, normalized color $r g b$ is computed as follows:

$$
\begin{aligned}
& r=R /(R+G+B) \\
& g=G /(R+G+B) \\
& b=B /(R+G+B) .
\end{aligned}
$$

However, this (nonlinear) color transformation has a serious drawback, as it becomes unstable for some $R G B$ sensor values. Particularly, $r g b$ is undefined at the black point $(R=G=B=$ 0 ) and is unstable near this singular point. As a consequence, a small perturbation for dark (low-intensity) sensor values (e.g., due to sensor noise) will cause a significant jump in the transformed values.

In Fig. 9(b), which is the normalized $r g b$ color transformation of Fig. 9(a), the unstable invariant values are clearly visible at the bottom of the red ball. The presence of these unstable invariant values violate the assumed Gaussian observation model. Fig. 9(c)-(e) shows the segmentation results for $K=6$ for the normalized color image of Fig. 9(b) with ICM, SimF, and MeanF, respectively. In Fig. 9(f), we show the corresponding results with our approach. All figures show a reconstruction of the normalized $r g b$ image, with pixel values in the final result corresponding to the estimated mean of their assigned Gaussian component. The results show that our approach produces more homogeneous regions than other algorithms and consequently a better segmentation.

\section{CONCLUSION}

We proposed a graphical model and a novel EM algorithm for Markov-based image segmentation. The proposed model postu- lates that the unobserved pixel labels are generated by prior distributions that have similar parameters for neighboring pixels. The proposed EM algorithm performs iterative bound optimization of a penalized log-likelihood of this model. The derived EM equations are similar to the standard (unconstrained) EM algorithm, with the only difference that a "smoothing" step is interleaved between the E- and the M-step, that couples the posteriors of neighboring pixels in each iteration. Compared to the other MRF-based algorithms for segmentation, we note that our algorithm enjoys a simple implementation and demonstrates competitive performance in terms of speed and solution quality.

\section{ACKNOWLEDGMENT}

The authors would like to thank K. Blekas, A. T. Cemgil, N. Galatsanos, I. Patras, and J. J. Verbeek for their valuable comments and N. Peyrard for making the implementation of MeanF, SimF, and ICM methods publicly available.

\section{REFERENCES}

[1] S. Geman and D. Geman, "Stochastic relaxation, Gibbs distributions, and the Bayesian restoration of images," IEEE Trans. Pattern Anal. Mach. Intell., vol. PAMI-6, no. 3, pp. 721-741, Mar. 1984.

[2] J. Besag, "On the statistical analysis of dirty pictures," J. Roy. Statist. Soc. Ser. B (Methodological), vol. 48, no. 3, pp. 259-302, 1986.

[3] R. Chellappa and A. Jain, Markov Random Fields: Theory and Application. Boston, MA: Academic, 1993.

[4] S. Z. Li, Markov Random Field Modeling in Computer Vision. London, U.K.: Springer-Verlag, 2001.

[5] M. V. Ibanez and A. Simo, "Parameter estimation in Markov random field image modeling with imperfect observations: A comparative study," Pattern Recognit. Lett., vol. 24, no. 14, pp. 2377-2389, 2003.

[6] D. Geman and B. Gidas, Image Analysis and Computer Vision. Washington, DC: National Academy Press, 1991, ch. 2, pp. 9-36.

[7] J.-M. Laferte, P. Perez, and F. Heitz, "Discrete Markov image modeling and inference on the quadtree," IEEE Trans. Image Process., vol. 9, no. 3, pp. 390-404, May 2000.

[8] J.-N. Provost, C. Collet, P. Rostaing, P. Perez, and P. Bouthemy, "Hierarchical Markovian segmentation of multispectral images for the reconstruction of water depth maps," Comput. Vis. Image Underst., vol. 93, no. 2, pp. 155-174, 2004.

[9] R. Fjortoft, Y. Delignon, W. Pieczynski, M. Sigelle, and F. Tupin, "Unsupervised classification of radar images using hidden Markov chains and hidden Markov random fields," IEEE Trans. Geosci. Remote Sens., vol. 41, no. 3, pp. 675-686, Mar. 2003.

[10] M. Robinson, M. Azimi-Sadjadi, and J. Salazar, "A temporally adaptive classifier for multispectral imagery," IEEE Trans. Neural Netw., vol. 15, no. 1, pp. 159-165, Jan. 2004.

[11] J. L. Marroquin, E. A. Santana, and S. Botello, "Hidden Markov measure field models for image segmentation," IEEE Trans. Pattern Anal. Mach. Intell., vol. 25, no. 11, pp. 1380-1387, Nov. 2003.

[12] S. C. Zhu, Y. Wu, and D. Mumford, "Filters, random fields and maximum entropy (FRAME): Towards a unified theory for texture modeling," Int. J. Comput. Vis., vol. 27, no. 2, pp. 107-126, 1998.

[13] G. McLachlan and D. Peel, Finite Mixture Models. New York: Wiley, Nov. 2000.

[14] C. Carson, S. Belongie, H. Greenspan, and J. Malik, "Blobworld: Image segmentation using expectation-maximization and its application to image querying," IEEE Trans. Pattern Anal. Mach. Intell., vol. 24, no. 8, pp. 1026-1038, Aug. 2002.

[15] A. Diplaros, T. Gevers, and N. Vlassis, "Skin detection using the EM algorithm with spatial constraints," in IEEE Int. Conf. Syst., Man Cybern., The Hague, The Netherlands, 2004, pp. 3071-3075.

[16] A. Dempster, N. Laird, and D. Rubin, "Maximum likelihood from incomplete data via the EM algorithm," J. Roy. Statist. Soc. Ser. B (Methodological), vol. 39, no. 1, pp. 1-38, 1977.

[17] R. Neal and G. Hinton, "A view of the EM algorithm that justifies incremental, sparse, and other variants," in Learning in Graphical Models, M. Jordan, Ed. Norwell, MA: Kluwer, 1998, pp. 355-368.

[18] W. Qian and D. M. Titterington, "Estimation of parameters in hidden Markov models," Philos. Trans. Roy. Soc. London A, Math. Phys. Sci., vol. 337, pp. 407-428, 1991. 
[19] J. Zhang, "The mean field theory in EM procedures for Markov random fields," IEEE Trans. Signal Process., vol. 40, no. 10, pp. 2570-2583, Oct. 1992.

[20] Y. Zhang, M. Brady, and S. Smith, "Segmentation of brain MR images through a hidden Markov random field model and the expectation-maximization algorithm," IEEE Trans. Med. Imag., vol. 20, no. 1, pp. 45-57, Jan. 2001.

[21] F. Forbes and N. Peyrard, "Hidden Markov random field model selection criteria based on mean field-like approximations," IEEE Trans. Pattern Anal. Mach. Intell., vol. 25, no. 9, pp. 1089-1101, Sep., 2003.

[22] J. Sun and D. Gu, "Bayesian image segmentation based on an inhomogeneous hidden Markov random field," in 17th Int. Conf. Pattern Recognit. (ICPR), 2004, vol. 1, pp. 596-599.

[23] G. Celeux, F. Forbes, and N. Peyrard, "EM procedures using mean field-like approximations for Markov model-based image segmentation," Pattern Recognit., vol. 36, no. 1, pp. 131-144, 2003.

[24] J. Besag, "Statistical analysis of non-lattice data," The Statistician, vol. 24, no. 3, pp. 179-195, 1975 .

[25] S. Sanjay-Gopal and T. J. Hebert, "Bayesian pixel classification using spatially variant finite mixtures and the generalized EM algorithm," IEEE Trans. Image Process., vol. 7, no. 7, pp. 1014-1028, Jul. 1998.

[26] K. Blekas, A. Likas, N. Galatsanos, and I. E. Lagaris, "A spatially-constrained mixture model for image segmentation," IEEE Trans. Neural Netw., vol. 16, no. 2, pp. 494-498, Mar. 2005.

[27] S. T. Roweis, L. K. Saul, and G. E. Hinton, "Global coordination of local linear models," in Advances in Neural Information Processing Systems 14 , T. G. Dietterich, S. Becker, and Z. Ghahramani, Eds. Cambridge, MA: MIT Press, 2002, pp. 889-896.

[28] J. J. Verbeek, N. Vlassis, and B. Kröse, "Self-organizing mixture models," Neurocomput., vol. 63, pp. 99-123, 2005.

[29] R. Xu and D. Wunsch, III, "Survey of clustering algorithms," IEEE Trans. Neural Netw., vol. 16, no. 3, pp. 645-678, May 2005.

[30] D. C. Stanford, "Fast automatic unsupervised image segmentation and curve detection in spatial point patterns," Ph.D. dissertation, Statistics Dept., Univ. Washington, Seattle, WA, 1999.

[31] D. C. Stanford and A. E. Raftery, "Approximate Bayes factors for image segmentation: The pseudolikelihood information criterion (PLIC)," IEEE Trans. Pattern Anal. Mach. Intell., vol. 24, no. 11, pp. 1517-1520, Nov. 2002

[32] D. Martin, C. Fowlkes, D. Tal, and J. Malik, "A database of human segmented natural images and its application to evaluating segmentation algorithms and measuring ecological statistics," in 8th IEEE Int. Conf. Comput. Vis., Jul. 2001, vol. 2, pp. 416-423.

[33] G. Healey, "Segmenting images using normalized color," IEEE Trans. Syst., Man, Cybern., vol. 22, no. 1, pp. 64-73, Jan./Feb. 1992.

[34] T. Gevers, "Adaptive image segmentation by combining photometric invariant region and edge information," IEEE Trans. Pattern Anal. Mach. Intell., vol. 24, no. 6, pp. 848-852, Jun. 2002.

[35] T. Gevers and A. W. M. Smeulders, "Color-based object recognition," Pattern Recognit., vol. 32, no. 3, pp. 453-464, 1999.

[36] G. Dong and M. Xie, "Color clustering and learning for image segmentation based on neural networks," IEEE Trans. Neural Netw., vol. 16, no. 4, pp. 925-936, Jul. 2005.

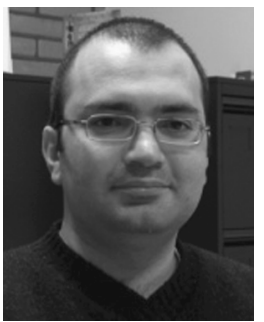

Aristeidis Diplaros (S'05-A'06) received the diploma in electronic and computer engineering from the Technical University of Crete, Crete, Greece, in 2001. He is currently working towards the Ph.D. degree in the Faculty of Science, the University of Amsterdam, Amsterdam, The Netherlands.

His research interests include computer vision and image retrieval.

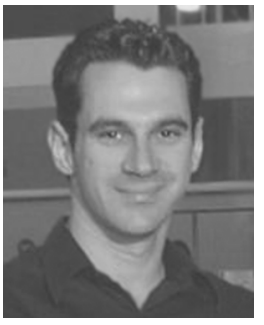

Nikos Vlassis received the M.Sc. degree in electrical and computer engineering in 1993 and the Ph.D. degree in artificial intelligence in 1998 from the National Technical University of Athens, Athens, Greece.

In 1999, he was a Visiting Researcher at the Electrotechnical Laboratory (currently AIST), Tsukuba, Japan, with a scholarship from the Japan Industrial Technology Association (MITI). In 2000, he joined the Informatics Institute, the University of Amsterdam, Amsterdam, The Netherlands, as an Assistant Professor. Since 2007 he has been an Assistant Professor at the Technical University of Crete, Hania, Crete, Greece. He is the coauthor of more than 80 papers in the fields of machine learning, robotics, multiagent systems, and vision.

Dr. Vlassis received the Dimitris Chorafas Foundation Prize (Luzern, Switzerland) for young researchers in engineering and technology, in 1998. He received the Best Scientific Paper Award in RoboCup-05 and his robot soccer team won the world championship in RoboCup-03.

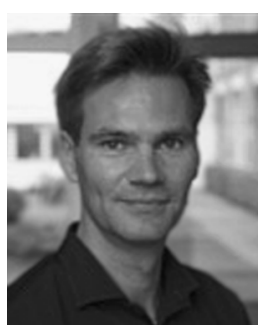

Theo Gevers (M'02) received the Ph.D. degree in computer science from the Faculty of Science, the University of Amsterdam, Amsterdam, The Netherlands, in 1996.

$\mathrm{He}$ is an Associate Professor of Computer Science and a Teaching Director of the graduate degree of artificial intelligence at the University of Amsterdam, Amsterdam, The Netherlands and an ICREA Research Professor at the Computer Vision Center (UAB), Barcelona, Spain. He has published over 100 papers on color image processing, image retrieval, and computer vision. His main research interests are in the fundamentals of content-based image retrieval, color image processing, and computer vision, specifically in the theoretical foundation of geometric and photometric invariants.

Mr. Gevers was the Co-Chair of various conferences and a Guest Editor of different special issues. He was the Program Committee Member of a large number of conferences, and the Invited Speaker at major conferences. He is a Lecturer of postdoctoral courses given at various conferences. 